\title{
El gest com a facilitador i precursor del desenvolupament del llenguatge
}

\author{
Esteve-Gibert, N.1, Igualada, A. ${ }^{1}$, Prieto, P. $^{2,3}{ }^{*}$ \\ ${ }^{1}$ Estudis de Psicologia i Educació, Universitat Oberta de Catalunya. \\ ${ }^{2}$ Institució Catalana de Recerca i Estudis Avançats, ICREA. \\ 3Departament de Traducció i Ciències del Llenguatge, Universitat Pompeu Fabra.
}

nesteveg@uoc.edu

aigualada@uoc.edu

pilar.prieto@upf.edu

Recepció: 14/05/2019, acceptació: 01/10/2019

Resum: Els estudis sobre desenvolupament del llenguatge típicament s'han centrat en l'anàlisi de la parla. Tanmateix, hi ha altres elements comunicatius que són una peça fonamental en la comunicació i cognició humanes: els gestos. Aquests elements visuals estan íntimament integrats amb la parla des del punt de vista temporal i des del punt de vista semanticopragmàtic, i fan que es pugui parlar del llenguatge i la comunicació com uns fets multimodals. En aquest article posem a l'abast dels lectors un resum dels estudis més recents sobre adquisició del llenguatge des d'una perspectiva multimodal, tot $\mathrm{i}$ emfasitzant el rol dels gestos com a facilitadors i precursors del llenguatge no només en les etapes més primerenques sinó també en etapes de desenvolupament pragmàtic més tardà. Els estudis demostren que el gest és un component central de l'adquisició del llenguatge i que actua com a precursor i predictor de l'aprenentatge del vocabulari, de la sintaxi, així com del desenvolupament pragmàtic i discursiu. Finalment apuntem les àrees de recerca més actuals i innovadores i el possible impacte d'aquesta recerca en l'àmbit de la rehabilitació del llenguatge.

Paraules clau: gest, modalitat gestual, adquisició lingüística inicial, precursors del llenguatge, comunicació multimodal

\section{El gesto como facilitador y precursor del desarrollo del lenguaje}

Resumen: Los estudios sobre desarrollo del lenguaje típicamente se han centrado en el análisis del habla. Sin embargo, hay otros elementos comunicativos que son una pieza fundamental en la comunicación y cognición humanas: los gestos. Estos elementos visuales están íntimamente integrados con el habla desde el punto de vista temporal y desde el punto de vista semanticopragmàtic, y hacen que se pueda hablar del lenguaje y la comunicación como unos hechos multimodales. En este artículo ponemos al alcance de los lectores un resumen de los estudios más recientes sobre adquisición del lenguaje desde una perspectiva multimodal, aunque enfatizando el rol de los gestos como facilitadores y precursores del lenguaje no sólo en las etapas más tempranas sino también en etapas de desarrollo pragmático más tardío. Los estudios demuestran que el gesto es un componente central de la adquisición del lenguaje y que actúa como precursor y predictor del aprendizaje del vocabulario, de la sintaxis, así como del desarrollo

\footnotetext{
${ }^{*}$ Els autors apareixen en ordre alfabètic.
} 
pragmático y discursivo. Finalmente apuntamos las áreas de investigación más actuales e innovadoras y el posible impacto de esta investigación en el ámbito de la rehabilitación del lenguaje.

Palabras clave: gesto, modalidad gestual, adquisición lingüística inicial, precursores del lenguaje, comunicación multimodal.

\section{Gesture as a facilitator and precursor of language development}

Abstract: Language development studies have typically focused on speech analysis. However, there are other communicative elements that are a fundamental piece in human communication and cognition: gestures. These visual elements are intimately integrated with speech from a temporal point of view and from a semanticopragmatic point of view, and make it possible to speak of language and communication as multimodal facts. In this article we provide readers with a summary of the most recent studies on language acquisition from a multimodal perspective, although emphasizing the role of gestures as facilitators and precursors of language not only in the earliest stages but also in later stages of pragmatic development. Studies show that gesture is a central component of language acquisition and that it acts as a precursor and predictor of vocabulary learning, syntax, pragmatic and discursive development. Finally, we point out the most current and innovative areas of research and the possible impact of this research in the field of language rehabilitation.

Keywords: gesture, gestural modality, initial linguistic acquisition, language precursors, multimodal communication.

\section{INTRODUCCIÓ}

Quan parlem fem servir gestos que acompanyen la parla. Aquests gestos moltes vegades complementen i aclareixen allò que estem dient. Per exemple, emprem sovint el gest d'assenyalar per aclarir que estem parlant de determinades persones, llocs o objectes en el context discursiu. En català també emprem gestos d'assentiment amb el cap quan estem d'acord amb el nostre interlocutor, expressions facials d'incredulitat com ara arrufar les celles quan no hi estem d'acord i expressions de sorpresa com ara aixecar les celles i obrir la boca. I sovint també fem servir gestos rítmics amb les mans i moviments de cap per emfasitzar diferents parts del discurs i focalitzar-les. Entenem per gest qualsevol moviment de les mans, del cap, del cos que acompanya la parla i que té una intenció comunicativa. A més, en parlar també produïm moviments amb la cara, o expressions facials, que complementen o modifiquen allò que expressem amb la parla. La Figura 1 mostra alguns exemples d'aquests gestos i expressions facials. 

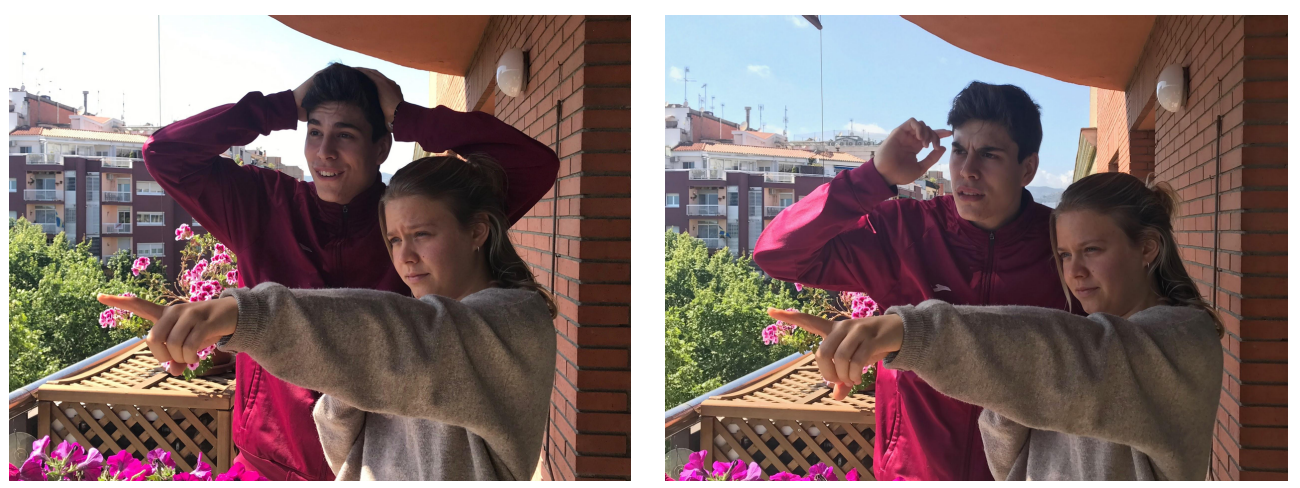

FIGURA 1. Imatges que mostren el gest d'assenyalar i l'expressió facial de sorpresa de l'interlocutor (esquerra), així com el mateix gest d'assenyalar i l'expressió facial d'incredulitat de l'interlocutor (dreta).

El contingut comunicatiu del discurs és, doncs, una combinació del que expressem i comprenem a través de la modalitat oral (la parla) i de la modalitat visual (els gestos i les expressions facials). No es pot entendre l'una sense l'altra i per això és necessari adoptar una visió multimodal del llenguatge que analitzi de manera integrada les dues modalitats i entengui que els gestos actuen com a elements lingüístics i comunicatius amb un valor comparable al de les paraules.

Un dels investigadors pioners en el ressorgiment de l'estudi de la gestualitat en les ciències cognitives actuals, David McNeill, afirma que els gestos i la parla formen un sistema totalment integrat tant des del punt de vista temporal com de significat (McNeill 1992). La integració temporal de gest i parla s'esdevé perquè els moviments corporals se sincronitzen amb les síl-labes acústicament més prominents (i alhora més rellevants) del discurs, demostrant que els patrons de producció gestual es coordinen sobretot en l'estructura prosòdica. Així, les parts més prominents dels gestos es coordinen temporalment amb posicions prominents de la parla (les síllabes amb accent tonal), i els inicis i finals dels moviments gestuals coincideixen amb els límits prosòdics dels enunciats (vg. Esteve-Gibert i col. 2017, Esteve-Gibert i Prieto 2013, Krivokapić 2014, Graziano i Gullberg 2018, entre d'altres). Des del punt de vista del significat, els gestos complementen la parla i codifiquen conjuntament un conjunt de significats tant lèxics com pragmàtics, aquests últims relacionats amb l'estructura de la informació, els actes de parla, el posicionament epistèmic o la cortesia. Recentment, s'ha demostrat que la connexió entre les dues modalitats és tan forta que certes àrees cerebrals estan implicades tant en la producció i la comprensió del gest com de la parla (Dimitrova, Chu, Wang, Özyürek i Hagoort 2016, Özyürek 2014).

Els infants adquireixen gestos i parla de forma conjunta i dinàmica al llarg dels primers anys de vida (per exemple, vg. Colletta i col. 2015, Sekine i Kita 2015), i el gest és un precursor del llenguatge, la comunicació i la cognició des de diferents punts de vista i en diferents etapes del desenvolupament. L'objectiu principal d'aquest l'article és posar a l'abast dels lectors les investigacions que en les darreres dècades han analitzat el paper precursor del gest en el desenvolupament del llenguatge, tant des del punt de vista temporal com semanticopragmàtic, en un període que va des del primer any de vida fins als 5 anys. La secció següent (secció 2) comenta el desenvolupament de la integració temporal entre gest i parla, així com en el valor predictiu que tenen aquestes combinacions gest-parla en l'adquisició d'habilitats lingüístiques que apareixen després en el desenvolupament. La secció 3 se centra en el desenvolupament de la integració entre 
gest i parla a nivell de significat, començant pel rol del gest com a precursor i predictor del vocabulari (secció 3.1), de la sintaxi (secció 3.3), de la intencionalitat comunicativa (secció 3.3.1), així com de la pragmàtica i del discurs (secció 3.3.2). Finalment, l'apartat final (secció 4) resumeix l'evidència empírica explicada al llarg de l'article i valora l'aplicació d'aquesta recerca en un seguit d'intervencions multimodals en entorns educatius i de tractament del llenguatge.

\section{EL DESENVOLUPAMENT DE LA INTEGRACIÓ TEMPORAL ENTRE GEST I PARLA}

Quan els infants desenvolupen el llenguatge també comencen a utilitzar les dues modalitats, gest i parla, per comunicar-se amb el seu entorn i per entendre allò que el seu entorn els vol comunicar. Això es fa especialment evident en les etapes inicials de l'adquisició del llenguatge, ja que el llenguatge oral dels infants és més rudimentari i el fet de disposar de recursos gestuals augmenta substancialment les seves possibilitats de comunicar-se amb l'entorn. Sovint els adults comprenem què volen dir els infants no pas per la informació de la seva parla, sinó gràcies als gestos que utilitzen i per la situació contextual en què aquests ocorren. Per tant, entendre el llenguatge i la comunicació infantil implica necessàriament tenir en compte les habilitats gestuals dels infants, que es desenvolupen en paral-lel i de manera dinàmica amb els altres components lingüístics i comunicatius.

Els parlants combinen la parla i els gestos corporals de manera molt precisa en l'àmbit temporal: les sílllabes acústicament més prominents del discurs (les sílllabes amb un accent tonal) se sincronitzen amb les fases gestualment més prominents del moviment corporal (en un gest d'assenyalar, per exemple, la màxima extensió del braç i del dit índex) (vg. Esteve-Gibert i col. 2017, Esteve-Gibert i Prieto 2013, Krivokapić 2014, Graziano i Gullberg 2018, entre d'altres).

Els infants han de desenvolupar l'habilitat de combinar gest i parla seguint aquests patrons tan precisos de coordinació temporal. Els primers senyals de la combinació temporal del sistema oral $\mathrm{i}$ gestual s'observen en etapes molt inicials del desenvolupament: durant els primers mesos els infants comencen a agafar objectes amb les mans, i se'ls acosten a la boca per experimentar-hi (Iverson i Fagan 2004). És habitual que vocalitzin mentre tenen aquests objectes a les mans o la boca. Uns mesos després, algunes d'aquestes vocalitzacions primerenques es converteixen en balbotejos en forma de concatenació de (proto)síl-labes del tipus bababa o dada. Alhora, les habilitats gestuals evolucionen de tal manera que els infants comencen a moure els braços en moviments rítmics. Els infants solen produir aquests gestos rítmics mentre balbotegen, i els balbotejos amb millor qualitat acústica i prosòdica són els que els infants produeixen mentre gesticulen de manera rítmica (Ejiri 1998, Ejiri i Masataka 2001).

Des d'un punt de vista perceptiu, des de ben aviat els infants aprenen a detectar si els moviments manuals, corporals o facials dels adults estan ben sincronitzats amb la parla. Als 2-4 mesos d'edat, els nadons noten si els moviments dels llavis d'una persona que parla estan sincronitzats amb el so que senten (Kuhl i Meltzoff 1984, Patterson i Werker 2003), i amb 9 mesos ja saben detectar si un adult assenyala i parla alhora seguint les regles precises de sincronització (és a dir, si la part prominent del gest coincideix amb l'accent tonal de la parla) (Esteve-Gibert, Prieto i Pons 2015).

Cap als 10-12 mesos, els infants comencen a assenyalar elements del seu entorn. És habitual que aquests primers gestos d'assenyalament ocorrin sense cap vocalització, és a dir, els infants assenyalen o bé en silenci o potser dient alguna cosa com ara tata. En aquests moments, és habitual que els infants assenyalin alguna cosa i produeixin alguna 
vocalització, però sovint els dos comportaments (assenyalar i vocalitzar) no estan ben sincronitzats, no ocorren alhora dins l'acte comunicatiu sinó que primer fan una cosa i després l'altra (Butcher i Goldin-Meadow 2000). Aquesta falta de coordinació entre modalitats és habitual i, sobretot, provisional. Tan aviat com dominen i estabilitzen les diverses habilitats lingüístiques i comunicatives, els infants comencen a combinar gest i parla de manera precisa, i són capaços de fer-ho seguint els patrons de sincronització precisos que també fem servir els adults (Iverson i Thelen 1999, Rusiewicz i Esteve-Gibert 2018). Així, per exemple, als 15-18 mesos els infants ja solen assenyalar i parlar alhora de manera que la síl-laba acústicament prominent coincidirà en el temps amb el moment en què el braç estigui més estès (en termes gestuals, aquest moment culminant del gest s'anomena stroke o àpex). Aquesta capacitat de combinar les dues modalitats amb una finalitat comunicativa representa un salt qualitatiu en les capacitats dels infants, ja que els permet emfasitzar el seu referent d'interès a través de la modalitat oral $i$ visual.

És important que aquesta habilitat de produir i percebre el gest i la parla de manera sincronitzada sigui tan primerenca en el desenvolupament dels infants, ja que pot servir com a indicador predictiu de les habilitats lingüístiques i comunicatives dels infants. S'ha vist, per exemple, que els infants que més combinaven el gest d'assenyalament amb la parla en etapes prelèxiques són els que més vocabulari expressiu tenen als 18 mesos (Igualada, Bosch i Prieto 2015; Iverson i Goldin-Meadow 2005). També, la sincronització de gest d'assenyalament i la parla podria ser un marcador fiable per detectar deficiències cognitives i comunicatives en els infants, per exemple en casos d'autisme (Fitzpatrick i col. 2017, Heymann, Northrup, West, Parlade, Leezenbaum i Iverson 2018) o de dèficits d'atenció (Norouzi, Hossieni, i Solymani 2018, Slater i Tate 2018). El cert és que calen més estudis empírics per entendre completament els patrons de coordinació de gest i parla, i alhora sobre com la necessitat de transmetre significats i intencions comunicatives en el discurs interactua amb les capacitats motrius, orals i comunicatives que té l'infant en cada etapa del desenvolupament lingüístic.

\section{Els GESTOS COM A ELEMENTS PRECURSORS I PREDICTORS DEL DESENVOLUPAMENT DEL LLENGUATGE}

\subsection{El gest com a precursor $i$ predictor del desenvo- lupament lèxic}

Què ho fa que uns infants tinguin més o menys vocabulari que d'altres? Podem predir el fet que uns infants comencin a produir les primeres paraules abans que d'altres? Tal com hem explicat en la secció anterior, els infants comencen a assenyalar elements del seu entorn cap als 10-12 mesos, és a dir, abans de ser capaços de produir l'etiqueta lèxica d'aquell referent (Iverson i Goldin-Meadow 2005). Com que el gest d'assenyalament precedeix la producció de lèxic, es considera que és un precursor del vocabulari dels infants. Una altra qüestió és, a més, si aquest gest díctic no només precedeix en el temps el lèxic, sinó si també té una funció predictiva en el desenvolupament del vocabulari. És a dir, podem tenir indicis de la capacitat de desenvolupament del vocabulari a partir de l'anàlisi dels gestos d'assenyalament que produeixen els infants uns mesos abans? En aquesta secció desgranarem aquestes qüestions.

Un estudi recent de McGillion i col. (2017) mostra que el desenvolupament del gest d'assenyalament no és, en si, un predictor de l'inici del vocabulari, és a dir, que no per començar a assenyalar abans, es comença a parlar abans. L'inici del gest d'assenyalament, doncs, no prediu l'inici del desenvolupament lèxic. Ara bé, el que sí s'ha vist és que si un infant comença a assenyalar abans, això tindrà conseqüències en les seves habilitats 
lèxiques en etapes posteriors del desenvolupament. En aquest mateix estudi, McGillion i col. mostren que si els infants de 10-12 mesos produeixen molts gestos d'assenyalament, aleshores quan tenen 18 mesos també entenen el significat de més paraules. Així doncs, la producció dels gestos d'assenyalament sí que té influència en el vocabulari receptiu (de comprensió) de l'infant. Pel que fa al vocabulari expressiu, també hi ha estudis que mostren la relació entre la quantitat de gestos d'assenyalament i la quantitat de vocabulari expressiu. D'una banda, s'ha demostrat que si un infant assenyala un objecte determinat, uns mesos més tard és molt probable que sàpiga produir l'etiqueta lèxica d'aquell objecte (Iverson i Goldin-Meadow 2005), i que els infants de 14-18 mesos que més assenyalaven són precisament els que tenen un vocabulari expressiu més gran en etapes preescolars posteriors (Rowe i Goldin-Meadow 2009a, 2009b).

Els motius que expliquen perquè els infants que produeixen gestos d'assenyalament amb més quantitat i de manera més primerenca són els que tenen després més vocabulari expressiu i receptiu, poden ser diversos. D’una banda, pot ser una qüestió d'habilitats lingüístiques i cognitives inherents als infants. No podem oblidar, però, que el llenguatge és un fet social, i que els infants assenyalen en un context d'interacció en què la gran majoria de vegades hi ha un adult al costat disposat a continuar l'intercanvi comunicatiu. És molt possible, doncs, que els infants que més assenyalin també afavoreixin l'inici de més intercanvis comunicatius i de tipus més complex, on l'infant i el receptor acaben formant part d'una situació d'atenció conjunta amb un objectiu comú (Heller i Rohlfing 2017). Quan un infant assenyala, els adults del seu voltant li diran el nom d'aquell objecte o esdeveniment, i sovint complementaran l'intercanvi comunicatiu amb molta més informació lingüística. Això pot afavorir que aquests infants rebin també més input lèxic i, per tant, acabin tenint un vocabulari més ric. En aquest supòsit, doncs, el que seria important del gest d'assenyalament seria, per tant, la quantitat i qualitat d'intercanvis comunicatius que generen al seu voltant. El cert és que encara no hi ha prou evidència científica per determinar si la importància del gest d'assenyalament es deu a un o altre motiu, i la discussió continua oberta (vg. Donnellan, Bannard, McGillion, Slocombe i Matthews 2019, Matthews, Behne, Lieven i Tomasello 2012).

\subsection{El gest com a precursor i predictor del desenvo- lupament sintàctic}

El desenvolupament gestual s'ha estudiat àmpliament al llarg del primer any de vida en relació amb el desenvolupament lèxic. Ara bé, l'ús de la modalitat gestual és també un factor relacionat amb l'habilitat sintàctica (o l'habilitat per combinar paraules)? La recerca ha demostrat que sí. En primer lloc, s'ha vist que la capacitat de combinació de paraules en la modalitat oral va precedida per una etapa durant la qual els infants combinen conceptes diferenciats en la modalitat oral i la gestual. Aquestes combinacions s'anomenen combinacions suplementàries (en anglès, supplementary combinations). Un exemple d'ús suplementari d'un gest es produeix quan un infant produeix la paraula vull mentre assenyala una joguina (gest díctic), o produeix la paraula mama alhora que fa el gest "vine" (gest representacional). Aquest ús de gestos representacionals i díctics amb funció suplementària s'ha descrit caps als 14-18 mesos, un cop el infants són capaços de produir conjuntament el mateix concepte en les dues modalitats (oral i gestual) (GoldinMeadow i Butcher 2003; Iverson i Goldin-Meadow 2005; Iverson, Capirci, Volterra i Goldin-Meadow 2008). Özçalişkan i Goldin-Meadow (2005) van observar longitudinalment les produccions multimodals d'infants als 14, 18 i 22 mesos i els resultats van mostrar que el nombre de combinacions suplementàries anava augmentant amb l'edat. En segon lloc, els resultats van mostrar que la capacitat de produir gestos amb 
valor suplementari prediu l'ús de construccions corresponents en la modalitat verbal en etapes posteriors. Per exemple, la producció de dos arguments en combinació suplementària (dir mama + gesticular sofà assenyalant-lo amb un gest díctic) als 18 mesos prediu la producció oral de formacions com mama cadira als 22 mesos). A més, aquest rol predictiu es va observar per al desenvolupament de frases amb més complexitat sintàctica. Així, la producció de combinacions de tres arguments (dir aquí mama + gest de bossa) als 18 mesos prediu aquí mama gos en la modalitat oral als 22 mesos. També, la combinació de predicat amb un altre argument (dir cap + gesticular rentar) als 18 mesos prediu formacions com bebè dorm en la modalitat oral als 22 mesos (Özçalişkan i Goldin-Meadow 2005). Per tant, veiem que el recurs gestual de l'assenyalament permet als nens produir significats cada vegada més complexos en un moment del desenvolupament en el qual encara no són capaços de produir-los en la modalitat verbal.

La recerca sobre gestos també ha estudiat si l'ús dels gestos pot influir en la comprensió sintàctica, és a dir, la comprensió de frases amb diferents graus de complexitat sintàctica. Theakston, Coates i Holler (2014) van demostrar que els nens de 3 i 4 anys eren capaços de reconèixer molt millor l'agent o el pacient d'una acció en construccions sintàctiques complexes quan es realitza un gest de manera simultània a la paraula. A aquest estudi van fer el gest d'assenyalar a l'aire amb la mà a diferents zones de l'espai gestual, assenyalaven la zona dreta durant la producció oral d'un rol temàtic seguit d'un gest a la zona esquerra per fer referència a l'altre rol temàtic. Com ara, a una construcció d'objecte clivellada del tipus «Va ser a la guineu, a qui va espantar el gos» ('It was the fox that the dog scared'), quan l'element guineu anava associat a un gest a la dreta, indicava la funció de pacient, i s'indicava la funció d'agent quan l'element gos anava acompanyat d'un gest a l'esquerra de l'espai gestual.

L'evidència empírica presentada en aquest apartat demostra que els gestos no només influeixen i estan relacionats amb la producció i la comprensió de la sintaxi, sinó que la producció de gestos també prediu el seu desenvolupament posterior en la modalitat oral. La Figura 2, extreta de Volterra, Caselli, Capirci i Pizzuto (2005), mostra l'evolució de les modalitats oral i gestual (gestos díctics i representacionals) dels 9 als 24 mesos. Mentre que la modalitat gestual domina sobre la modalitat oral abans dels 14 mesos, a partir dels 14 mesos la modalitat gestual i oral mantenen un ús paral-lel i es pot observar com la modalitat gestual té un rol rellevant en el desenvolupament de les funcions lingüístiques posteriors expressada a través de la combinació de dues paraules als 24 mesos. 


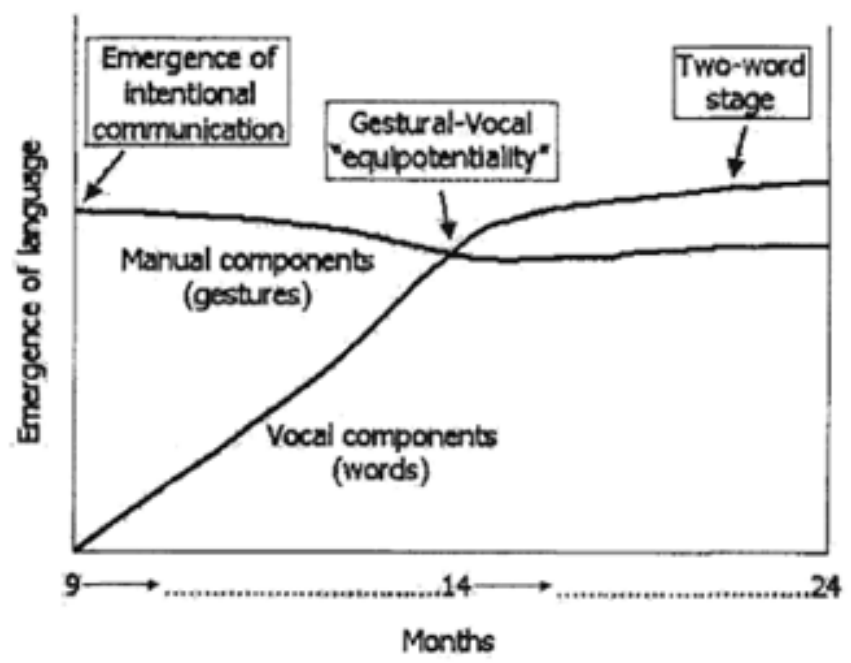

FIGURA 2. Imatge extreta de Volterra, Caselli, Capirci i Pizzuto (2005) que mostra la permanència en l'ús de la modalitat gestual un cop ha aparegut el llenguatge oral.

\subsection{El gest com a predictor de les habilitats pragmàtiques}

\subsubsection{El desenvolupament primerenc de les intencions comunicatives}

Els infants es mouen i vocalitzen des de les primeres setmanes de vida. Tot i que els adults intentem atorgar significat lingüístic a aquests primers moviments i sons, el cert és que són actes reflexos que els infants produeixen com a resultat d'algun estat intern i sense intencionalitat comunicativa. Per exemple, ploren perquè tenen fred o vocalitzen $\mathrm{i}$ riuen quan veuen alguna cosa que els provoca benestar. No és fins als 9-10 mesos d'edat que els infants són capaços de vocalitzar i gesticular amb intenció comunicativa, és a dir, amb la voluntat de provocar algun efecte en el seu entorn, de canviar-lo. Un cop els infants desenvolupen l'habilitat de comunicar-se de manera intencional amb el seu entorn (ja sigui amb gestos o amb parla), i un cop entenen que els sons i els gestos que produeixen els adults també tenen un objectiu comunicatiu, es considera que s'han establert les bases comunicatives per a l'adquisició del llenguatge.

Hi ha dos elements relacionats amb la gestualitat que permeten veure si l'infant desenvolupa de manera típica la intencionalitat comunicativa. D’una banda, el fet que siguin capaços de mirar allà on els altres miren o de mirar on els altres assenyalen. De l'altra, la capacitat de produir i comprendre gestos d'assenyalament. A continuació explicarem amb més detall aquests dos elements.

Els nadons acabats de néixer no entenen que quan mirem o assenyalem un element del nostre entorn ho fem per indicar el focus d'interès, i que si ens girem i/o dirigim la nostra mirada cap a un altre referent, volem que ells també mirin cap allà. Això comença a canviar als 2-3 mesos: els infants comencen a entendre que girar el cap envers algun punt del nostre entorn vol dir que ens interessa aquell punt del nostre entorn; a partir dels 6 mesos comencen a ser capaços de seguir el focus de la mirada dels altres, i cap als 9 mesos comencen a mirar allà on els altres assenyalen (Tenenbaum, Sobel, Sheinkopf, Malle i Morgan 2015). És important fer un seguiment d'aquests comportaments perquè s'ha demostrat que la capacitat dels infants de seguir el focus d'interès de la mirada o del 
gest d'assenyalament dels altres prediu el vocabulari que tindran els infants en etapes més avançades del seu desenvolupament, i les capacitats d'inferència pragmàtica $o$ Teoria de la Ment (és a dir, posar-se al lloc dels altres i imaginar-se allò que estan pensant) (Baron-Cohen 1994, Morales, Mundy i Rojas 1998).

La capacitat de comprendre la intencionalitat que tenen els gestos d'assenyalament és un altre indicador fiable de les habilitats comunicatives de l'infant. Quan assenyalem podem tenir una intenció declarativa (l'objectiu és l'atenció de l'altre i el referent assenyalat és el focus de l'interès informatiu) o una intenció de petició o imperativa (l'objectiu és el referent assenyalat i l'interlocutor és una eina per aconseguir-ho). S'ha vist que als 12-18 mesos els infants ja entenen si un gest d'assenyalar té intenció declarativa o imperativa (Aureli, Perucchini i Genco 2009, Behne, Liszkowski, Carpenter i Tomasello 2012, Camaioni, Perucchini, Bellagamba i Colonnesi 2004), fins i tot quan el context és ambigu i només la forma de la mà i la prosòdia de la parla són els únics possibles indicadors d'aquesta intenció (Esteve-Gibert, Prieto i Liszkowski 2017, EsteveGibert i Prieto 2018).

S'ha demostrat que els infants amb Trastorn de l'Espectre Autista (TEA) tenen dificultats per comprendre la intenció declarativa dels gestos d'assenyalament però tenen una comprensió típica dels gestos d'assenyalament amb intenció imperativa (Camaioni, Perucchini, Muratori, Parrini, i Cesari 2003). Com que els infants amb desenvolupament típic comprenen el gest d'assenyalament amb intenció declarativa entre els 12 i els 18 mesos d'edat, la comprensió del gest d'assenyalament pot constituir un possible indici per detectar de forma primerenca el TEA, que normalment es diagnostiquen més tard en el desenvolupament dels infants.

\subsubsection{El desenvolupament de les habilitats pragmàtiques més tardanes}

Els gestos també tenen un rol crucial en el desenvolupament de les habilitats pragmàtiques en etapes posteriors, i més concretament en el desenvolupament referencial (introducció d'elements nous en el discurs), el posicionament epistèmic (detecció de la incertesa, la incredulitat, etc.) i, en general, en les habilitats discursives (Andrés-Roqueta 2018, Matthews 2014). De fet, estudis recents han demostrat que, de manera similar a altres components del llenguatge, les habilitats multimodals prèvies tenen un paper important de suport en el desenvolupament d'aquestes habilitats pragmàtiques (Armstrong, Esteve-Gibert, Hübscher, Igualada i Prieto 2018, Hübscher, Esteve-Gibert, Igualada i Prieto 2017). Els nens de 3 a 5 anys es beneficien de la presència dels marcadors multimodals (expressions facials i prosòdia) a l'hora de detectar la incredulitat dels interlocutors (Armstrong i col. 2018) i el grau de certesa del parlant sobre la informació expressada (Hübscher i col. 2017), i també saben emprar ells mateixos els elements multimodals per expressar aquests significats pragmàtics complexos (Hübscher, Garufi i Prieto 2019, Hübscher, Vincze i Prieto 2019). Així, els nens d'aquesta edat comprenen i utilitzen els marcadors gestuals i prosòdics de la incertesa i la incredulitat abans que es desenvolupi la comprensió i producció d'aquesta informació pragmàtica amb marcador lèxics i morfosintàctics. Aquests estudis apunten a un efecte impulsor de la multimodalitat en la comprensió de significats pragmàtics epistèmics com ara la incredulitat $o$ la incertesa.

Altres àrees de la pragmàtica estudiades des de la perspectiva multimodal han estat l'ús de la referencialitat per introduir elements nous en el discurs. Segons So i Lim (2012), quan els adults acompanyen un element nou en el discurs amb un gest, els nens de 4 anys ho detecten i responen a aquella interacció amb més freqüència. Això també s'ha observat 
en les produccions dels mateixos nens, que als 5 anys fan servir més gestos per introduir referents nous que per referir-se a elements mencionats prèviament en el discurs, sobretot quan són nens bilingües i s'estan comunicant en la seva llengua menys dominant (So, Lim i Tan 2012).

Els gestos rítmics (beat gestures, en anglès) són gestos no representacionals associats a les parts prosòdicament prominents de la parla i que serveixen per marcar el focus i l'estructura informativa del discurs. Els nens en l'etapa infantil comencen a emprar aquests tipus de gestos per expressar i desenvolupar habilitats narratives més complexes. Mathew, Yuen i Demuth (2017) van mostrar que mentre expliquen una història els nens entre 5 i 7 anys produeixen gestos rítmics amb les mans de manera molt semblant als adults per marcar les part rellevants del discurs. També s'ha demostrat que els nens d'aquesta edat fan servir els gestos rítmics amb les mans (i també amb el cap) abans que els indicadors prosòdics de la parla per marcar les estructures informatives rellevants del seu discurs (Esteve Gibert, Lœvenbruck, Dohen i D'Imperio 2019, Mathew, Yuen i Demuth 2017). Quant a la comprensió, s'ha demostrat que els nens de 3 a 4 anys es beneficien de la funció focalitzadora dels gestos rítmics per millorar el record de paraules presentades en un context discursiu (Igualada, Esteve-Gibert i Prieto 2017). També s'ha demostrat que els nens de 5 i 6 anys produeixen estructures narratives més complexes quan s'entrenen en l'observació de narracions explicades amb gestos rítmics (VilàGiménez, Igualada i Prieto, 2019).

En línia amb aquests resultats, Demir, Levine i Goldin-Meadow (2015) han demostrat que l'ús primerenc dels gestos durant la producció de narracions als 5 anys prediu l'habilitat narrativa expressiva en edats posteriors. En aquest estudi longitudinal l'habilitat dels nens de 5 anys per produir gestos representacionals expressats des del punt de vista del personatge de la narració, com ara fer l'acció de llençar una pilota en comptes de definir la forma de la pilota amb un gest, va predir la complexitat oral posterior d'aquests mateixos infant.

En resum, tot un conjunt d'evidències demostra que la pragmàtica és una altra àrea del desenvolupament del llenguatge influenciada per la modalitat gestual, tant en l'expressió com en la comprensió. A més, de manera semblant al que hem vist en apartats anteriors, es corrobora la hipòtesi del desenvolupament previ de les competències pragmàtiques en la modalitat gestual.

\section{CONCLUSIONS}

En aquest article hem revisat una sèrie d'investigacions recents que demostren que, des dels seus inicis, el desenvolupament del llenguatge està íntimament lligat al desenvolupament del gest i que aquest últim té una funció precursora i de facilitació del llenguatge. Hem vist que, cap als nou mesos, i molt abans de produir les primeres paraules, els infants fan servir el dit o la mà per assenyalar, cosa que els permet atreure l'atenció dels pares o cuidadors vers un referent d'interès i iniciar o continuar un intercanvi comunicatiu. Precisament és en aquesta etapa primerenca de l'adquisició del llenguatge que la capacitat integració temporal entre els gestos i la parla actua com a precursora i predictora de l'adquisició del llenguatge (vg. secció 2). També hem vist que l'ús dels gestos díctics és un clar precursor del desenvolupament lèxic (secció 3.1), sintàctic (secció 3.2) i pragmàtic (secció 3.3). Mentre que la investigació s'ha centrat tradicionalment en l'important paper precursor del gest en l'adquisició primerenca del vocabulari i la morfosintaxi, menys estudis s'han concentrat en l'adquisició més tardana del llenguatge, i concretament de la pragmàtica. Tanmateix, també hem vist que en moments posteriors del desenvolupament els nens de 3 a 5 anys (a) continuen emprant 
els gestos díctics com a facilitadors de la referencialitat en el discurs; (b) fan servir expressions facials que actuen de precursors i potenciadors de les habilitats de producció $\mathrm{i}$ detecció de la incertesa i la incredulitat; i (c) fan servir gestos representacionals i també gestos rítmics que actuen com a precursors i potenciadors de les habilitats discursives. Així doncs, l'habilitat multimodal dels nens constitueix una habilitat comunicativa essencial per al desenvolupament no només del lèxic, sinó també de la sintaxi i la pragmàtica, així com un indicador clau en el període de la construcció del llenguatge al llarg dels primers anys de vida (per a un resum, vg. Esteve-Gibert i Guellaï 2018, Hübscher i Prieto 2019 i Igualada 2018).

L'evidència presentada en aquest article mostra que el desenvolupament del llenguatge des dels seus inicis és inherentment multimodal i que són precisament aquestes característiques multimodals del llenguatge les que actuen de facilitadors per al desenvolupament lingüístic dels nens. És important doncs que tant els pares com els educadors i cuidadors sàpiguen aprofitar aquest potencial de la comunicació gestual al llarg del desenvolupament, i sobretot durant el primer any de vida. En aquest sentit, l'eina web produïda en la nostra llengua, Abans de les primeres paraules (http://abansprimeresparaules.upf.edu/) -també en castellà i en anglès-, explica de forma didàctica a través de materials audiovisuals com els infants desenvolupen les habilitats comunicatives al llarg del primer any de vida, tot adoptant una visió multimodal que té molt en compte la comunicació gestual. També es donen consells per part d'experts sobre com es poden potenciar aquestes habilitats comunicatives. Per tal de fer apreciar aquestes capacitats comunicatives de l'infant, el web fa un repàs de les diferents habilitats multimodals al llarg del primer any de vida, que es comenten tant des del punt de vista de la percepció, de l'expressió com de la interacció (vg. Figura 3).

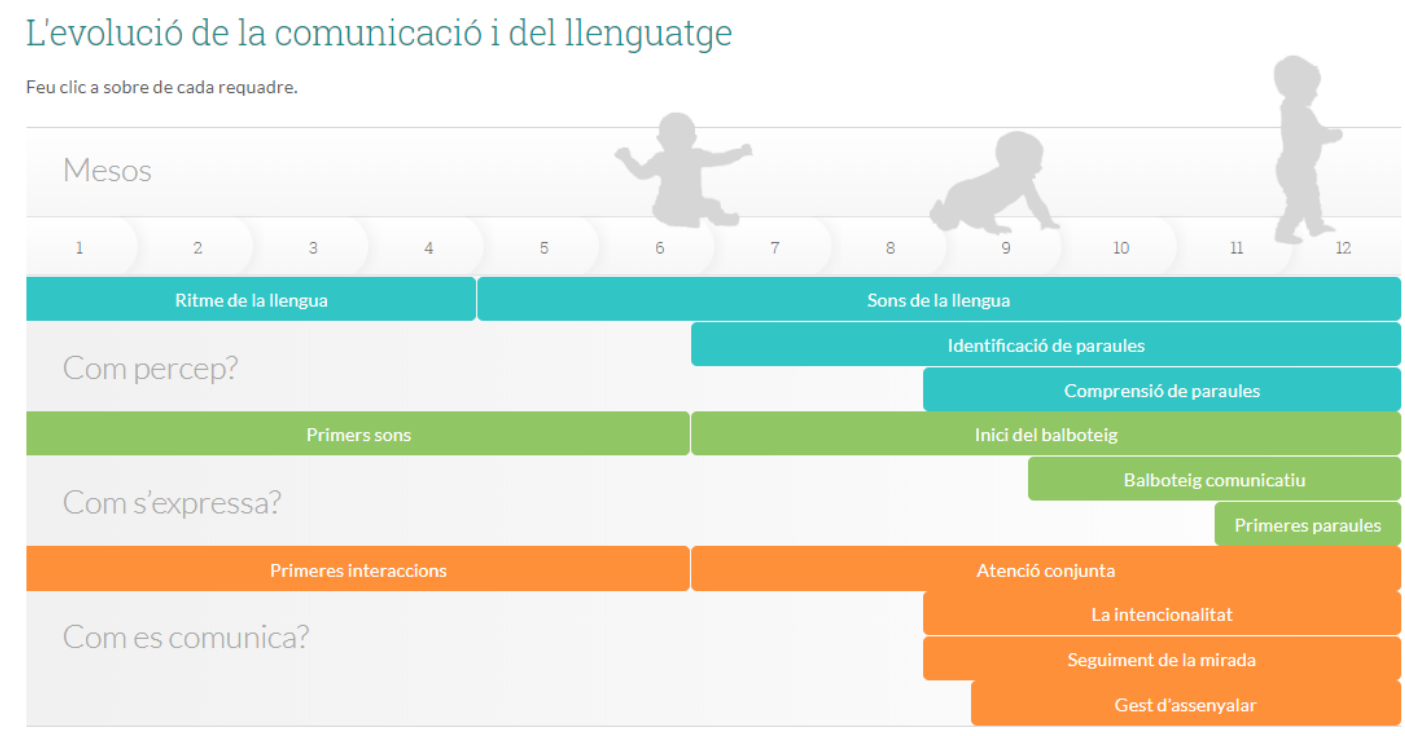

FIGURA 3. Evolució cronològica com evolucionen les habilitats comunicatives i

lingüístiques durant els primers 12 mesos de vida dels infants, extreta del web Abans de les primeres paraules.

Creiem que la funció precursora i predictora del gest hauria de constituir un pilar fonamental de suport a l'aprenentatge i el desenvolupament en els àmbits de l'educació i de la rehabilitació del llenguatge. En aquest sentit, no és estrany que s'hagi demostrat que els entrenaments multimodals que potencien la part no-verbal del llenguatge s'utilitzin amb èxit en entorns educatius i d'intervenció lingüística. Per exemple, com hem vist, l'estudi recent de Vilà-Giménez i col. (2019) ha obtingut un efecte positiu d'un 
entrenament multimodal amb gestos rítmics en les habilitats narratives i de fluïdesa de les narracions en un grup de nens de 5-6 anys. D'altra banda, un conjunt d'estudis han demostrat que les intervencions basades en habilitats d'imitació de gest amb nens amb trastorn de l'espectre autista (TEA) generen majors guanys en la taxa d'ús de la llengua (Ingersoll 2008, Ingersoll, Lewis i Kroman 2007).

En resum, creiem que atesa la seva importància és necessari incorporar de manera decidida una visió multimodal de la comunicació en l'estudi del desenvolupament del llenguatge. Tot i que encara estem lluny de tenir un panorama complet del desenvolupament infantil del gest i de la parla en diferents contextos comunicatius, un enfocament més holístic, on les diferents funcions i interaccions de la parla i el gest s'estudiïn conjuntament segur que enriquirà el camp i ens ajudarà a aplicar de manera més decidida els coneixements obtinguts als camps de l'educació i la logopèdia.

\section{REFERÈNCIES}

ANDRÉS-RoQueta, ClaRa (2018). «Desarrollo de la pragmática» APARICI AZNAR, MELINA; IguALAdA, AlFonso (eds.). El desarrollo del lenguaje y la comunicación en la infancia. Barcelona: Editorial UOC, 141-166.

Armstrong, Meghan; Esteve Gibert, Núria; Hübscher, Iris; Igualada, Alfonso; PRIETO, PILAR (2018). "Developmental and cognitive aspects of children's disbelief comprehension through intonation and facial gesture». First Language, 38(6), 596-616.

Aureli, Tiziana; Perucchini, PaOla; Genco, Maria (2009). «Children's understanding of communicative intentions in the middle of the second year of life». Cognitive Development, 24(1), 1-12.

BARON-COHEN, Simon (1994) «How to build a baby that can read minds: Cognitive mechanisms in mindreading». Cahiers de Psychologie Cognitive/Current Psychology of Cognition, 13, 513-552.

Behne, Tanya; Liszkowski, Ulf; Carpenter, Malinda; Tomasello, Michael (2012). «Twelve-month-olds' comprehension and production of pointing». The British Journal of Developmental Psychology, 30(3), 359-375.

Butcher, CyNThia; Goldin-MEadow, Susan (2000). «Gesture and the transition from one- to two- word speech. When hand and mouth come together». MCNEILL, DAVID (ed.). Language and gesture. New York: Cambridge University Press, 235-257.

Camaioni, luigia; Perucchini, Paola; Bellagamba, Francesca; Colonnesi, Cristina (2004). «The Role of Declarative Pointing in Developing a Theory of Mind». Infancy, 5(3), 291-308.

Camaioni, luigia; Perucchini, Paola; Muratori, F., Parrini, Barbara; Cesari, Alessia (2003). "The communicative use of pointing in autism: developmental profile and factors related to change». European Psychiatry, 18(1), 6-12.

Colletta, Jean-Marc; Guidetti, Michèle; Caprici, Olga; Cristilli, Carla; Demir, Oslem EZE; KunENe-Nicolas, RAMONa N.; LEvine, Susan (2015). «Effects of age and language on co-speech gesture production: An investigation of French, American, and Italian children's narratives». Journal of Child Language, 42(1), 122-145. DEMIR, ÖZLEM ECE; LeVINE, SusAn C.; Goldin-MEAdOW, Susan (2015). «A tale of two hands: Children's early gesture use in narrative production predicts later narrative structure in speech». Journal of Child Language, 42(3), 662-681.

Dimitrova, Diana; Chu, Mingyuan; Wang, Lin; Ozyurek, Asli; Hagoort, Peter (2016). «Beat that Word: How Listeners Integrate Beat Gesture and Focus in Multimodal Speech Discourse». Journal of Cognitive Neuroscience, 28(9), 1255-1269. 
Donnellan, Ed; Bannard, Colin; McGillion, Michelle, L.; Slocombe, Katie E.; MATTHEWS, DANIELLE (2019). «Infants' intentionally communicative vocalisations elicit responses from caregivers and are the best predictors of the transition to language: A longitudinal investigation on infants' vocalisations, gestures, and word production». Developmental Science (online first view).

EJIRI, KEIKO (1998). «Relationship between rhythmic behavior and canonical babbling in infant vocal development». Phonetica, 55, 226-237.

EJIRI, KeIKo; MASATAKA, NoBUo (2001). «Co-occurrence of preverbal vocal behavior and motor action in early infancy». Developmental Science, 4, 6-11.

Esteve-Gibert, Núria; Prieto, Pilar (2013). «Prosody signals the emergence of intentional communication in the first year of life: evidence from Catalan-babbling infants». Journal of Child Language, 40(5), 919-944.

Esteve-Gibert, Núria; Borràs-Comes, JoAn; Asor, Eli; Swerts, Marc; Prieto, Pilar (2017). «The timing of head movements: the role of prosodic heads and edges». Journal of the Acoustical Society of America, 141(6), 4727-4739.

ESteVE-Gibert, NúRIA; Guellaï, BAHIA (2018). «Prosody in the auditory and visual domains: A developmental perspective». Frontiers in Psychology, 9, 1-10.

Esteve Gibert, NÚRIa; LeVenbruck, HélÈne; Dohen, Marion; D’IMPerio, MariapaOla (2019). «Pre-schoolers use head gestures rather than duration or pitch range to signal narrow focus in French». Proceedings form the International Congress of Phonetic Sciences. Melbourne, Australia.

Esteve-Gibert, Núria; Prieto, Pilar (2018). «Early development of the prosodymeaning interface» PrIETO, PILAR; Esteve-Gibert, NúRIA (eds.). The Development of Prosody in First Language Acquisition. Amsterdam: John Benjamins, 227-246.

Esteve-Gibert, NÚRIA; Prieto, Pilar; Pons, Ferran (2015). «Nine-month-old infants are sensitive to the temporal alignment of prosodic and gesture prominences». Infant Behavior and Development, 38, 126-129.

Esteve-Gibert, Núria; Prieto, Pilar; Liszkowski, Ulf (2017). «Twelve-month-olds understand social intentions based on prosody and gesture shape». Infancy, 22(1), 108129.

FitzPatrick, Paula; Romero, Veronica; Amaral, Joseph L.; Duncan, Amie; Barnard, Holly; RichaRdSON, MichaEL J.; SCHMIDT, R. C. (2017). «Social Motor Synchronization: Insights for Understanding Social Behavior in Autism». Journal of Autism and Developmental Disorders, 47(7), 2092-2107.

Goldin-Meadow, Susan; Butcher, Cynthia (2003). «Pointing toward two-word speech in young children» Kita, Sotaro (ed.). Pointing: Where Language, Culture, and Cognition Meet. Mahwah, NJ: Erlbaum, 85-107.

Graziano, Maria; Gullberg, Marianne (2018). «When Speech Stops, Gesture Stops: Evidence From Developmental and Crosslinguistic Comparisons». Front Psychol, 9, 879.

Heymann, Perrine; Northrup, Jessie B.; West, Kelsey L.; Parlade, Meaghan P.; LEEZENBAUM, NinA B.; IVERSON, JANA M. (2018). «Coordination is key: joint attention and vocalisation in infant siblings of children with autism spectrum disorder». International Journal of Language and Communication Disorders, 53(5), 1007-1020.

HELLER, VIVIEN; ROHLFING, KATHARINA (2017). «Reference as an Interactive Achievement: Sequential and Longitudinal Analyses of Labeling Interactions in Shared Book Reading and Free Play». Frontiers in Psychology, 14(8), 139. 
Hübscher, Iris; Esteve-Gibert, Núria; Igualada, Alfonso; Prieto, Pilar (2017). «Intonation and gesture as bootstrapping devices in speaker uncertainty». First Language, 37(1), 24-41.

HÜbscher, Iris; Garufi, Martina; Prieto, Pilar (2019). «The development of polite stance in preschoolers: how prosody, gesture and body cues pave the way». Journal of Child Language, 46(5), 826-862.

HÜBSCHER, IRIS; PRIETO, PILAR (2019). «Gestural and prosodic development act as sister systems and jointly pave the way for children's sociopragmatic development». Frontiers in Psychology, 10.

HÜbscher, IRis; Vincze, Laura; Prieto, Pilar (2019). «Children's signaling of their uncertain knowledge state: prosody, face and body cues come first». Journal of Language, Learning and Development, 15(4), 366-389.

Igualada, Alfonso; Bosch, Laura; Prieto, Pilar (2015). «Language development at 18 months is related to communicative strategies at 12 months». Infant Behavior and Development, 39, 42-52.

Igualada, Alfonso; Esteve-Gibert, NÚRIA; Prieto, Pilar (2017). «Beat gestures improve word recall in 3-to 5-year-old children». Journal of Experimental Child Psychology, 156, 99-112.

Igualada, Alfonso (2018). «Desarrollo del gesto y del habla». APARici AZnAR, MELINA; IgUALADA, AlFONSO (eds.). El desarrollo del lenguaje y la comunicación en la infancia. Barcelona: Editorial UOC, 191-208.

INGERSOLL, BROOKE (2008). «The social role of imitation in autism: Implications for the treatment of imitation deficits». Infants and Young Children, 21, 107-119.

Ingersoll, Brooke; LeWis, ElizABETH; Kroman, Emily (2007). «Teaching the imitation and spontaneous use of descriptive gestures in young children with autism using a naturalistic behavioral intervention». Journal of Autism and Developmental Disorders, $37(8), 10$.

IVERSON, JANA M.; FAGAN, MARY K. (2004). «Infant vocal-motor coordination: precursor to the gesture-speech system?». Child Development, 75, 1053-1066.

IVERSON, JANA M; Goldin-MEADOW, SUSAN (2005). «Gesture paves the way for language development». Psychological Science, 16(5), 367-71.

Iverson, JANA M; THelen, Esther (1999). «Hand, mouth and brain: the dynamic emergence of speech and gesture». Journal of Consciousness Studies, 6, 19-40.

Iverson, Jana M.; CAPIrci, Olga; Volterra, Virginia; Goldin-Meadow, Susan (2008). «Learning to talk in a gesture-rich world: early communication of Italian versus American children». First Language, 28,164-81.

KRIVOKAPIĆ, JELENA (2014). «Gestural coordination at prosodic boundaries and its role for prosodic structure and speech planning processes». Philosophical Transactions of the Royal Society B: Biological Sciences, 369(1658).

Kuhl, Patricia K.; Meltzoff, Andrew N. (1984). «The intermodal representation of speech in infants». Infant Behavior and Development, 7, 361-381.

MCNEILL, DAVID (1992). «Hand and mind - What gestures reveal about thought». Chicago, IL: University of Chicago Press.

Mathew, Mili; Yuen, Ivan; Demuth, Katherine (2017). «Talking to the beat: Six-yearolds' use of stroke-defined non-referential gestures». First Language, 38, 1-18. 
Matthews, Danielle (ED.). (2014). «Pragmatic development in first language acquisition» (Vol. 10). John Benjamins Publishing Company.

Matthews, Danielle; Behne, Tanya; Lieven, Elena; Tomasello, Michael (2012). «Origins of the human pointing gesture: a training study». Developmental Science, 15(6), 817-829.

McGillion, Michelle; Herbert, Jane S.; Pine, Julian; Vihman, Marilyn; De Paolis, Rory; Keren-Portnoy, Tamar; Matthews, Danielle (2017). «What Paves the Way to Conventional Language? The Predictive Value of Babble, Pointing, and Socioeconomic Status». Child Development, 88(1), 156-166.

Morales, Michael; Mundy, Peter; Rojas, Jennifer (1998). «Following the direction of gaze and language development in 6-month-olds». Infant Behavior \& Development, 21(2), 373-377.

Norouzi, Ebrahim; Hossieni, Fatemeh; Solymani, Mehran (2018). «Effects of Neurofeedback Training on Performing Bimanual Coordination In-phase and Anti-phase Patterns in Children with ADHD». Applied Psychophysiology and Biofeedback, 43, 283.

OzÇALIŞKAN, SEYDA; GOLDIN-MEADOW, SusAn (2005). «Gesture is at the cutting edge of early language development». Cognition, 96(3), B101-13.

ÖZYÜREK, ASLI (2014). «Hearing and seeing meaning in speech and gesture: insights from brain and behaviour». Philosophical Transactions of the Royal Society of London. Series B, Biological Sciences, 369(1651), 20130296.

Patterson, Michelle L.; Werker, Janet F. (2003). «Two-month-old infants match phonemic information in lips and voice». Developmental Science, 6, 191-196.

Rowe, MEREDith. L; Goldin-MEAdow, SUSAN (2009a). «Early gesture selectively predicts later language learning». Developmental Science, 12(1), 182-187.

- - (2009b). «Differences in early gesture explain SES disparities in child vocabulary size at school entry». Science, 323(5916), 951-953.

Rusiewicz, Heather L.; Esteve-Gibert, Núria (2018). «Temporal coordination of prosody and gesture in the development of spoken language production». PRIETO, PILAR; ESTEVE-GiberT, NÚRIA (eds.). The Development of Prosody in First Language Acquisition. Amsterdam: John Benjamins, 103-124.

SEKINE, KAZUKI; KITA, SOTARO (2015). «The parallel development of the form and meaning of two-handed gestures and linguistic information packaging within a clause in narrative». Open linguistics, 1(1), 490-502.

Slater, Jessicas L.; Tate, MatThew C. (2018). «Timing Deficits in ADHD: Insights From the Neuroscience of Musical Rhythm». Frontiers in Computational Neuroscience, 12, 51.

So, Wing CheE; LIM, JiA-Yi (2012). «"What is this?” Gesture as a potential cue to identify referents in discourse». Applied Psycholinguistics, 33(2), 329-342.

So, Wing ChEe; LIM, JiA-YI; TAN, SEOK-Hui (2012). «Sensitivity to information status in discourse: Gesture precedes speech in unbalanced bilinguals». Applied Psycholinguistics, 35(2014), 71-95.

Tenenbaum, Elena J.; Sobel, David M.; Sheinkopf, Stephen J.; Malle, Bertram F.; MORGAN, JAMES L. (2015). «Attention to the mouth and gaze following in infancy predict language development». Journal of Child Language, 42(6), 1173-1190.

Theakston, Anna L.; CoATes, AnNA; Holler, Judith (2014). «Handling agents and patients: representational cospeech gestures help children comprehend complex syntactic constructions». Developmental Psychology, 50(7), 1973-1984. 
Vilà-GiméneZ, Ingrid; IGUALAdA, Alfonso; Prieto, Pilar (2019). «Observing storytellers who use rhythmic beat gestures improves children's narrative discourse performance». Developmental Psychology, 55(2), 250-262.

Volterra, Virginia; Caselli, Maria Cristna; Capirci, Olga; Pizzuto, Elena (2005). «Gesture and the emergence and development of language». Beyond nature-nurture: Essays in honor of Elizabeth Bates, 3, 40. 